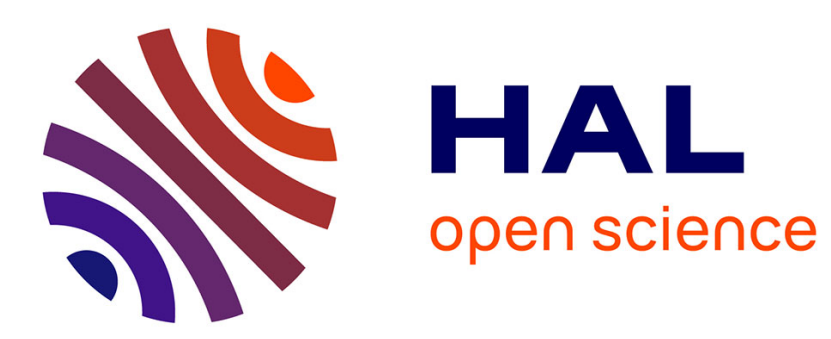

\title{
La destruction du patrimoine culturel à Alep : banalité d'un fait de guerre ?
}

Jean-Claude David, Thierry Boissière

\section{To cite this version:}

Jean-Claude David, Thierry Boissière. La destruction du patrimoine culturel à Alep : banalité d'un fait de guerre ?. Confluences Méditerranée , 2014, 89 (2), 10.3917/come.089.0163 . hal-01925452

\section{HAL Id: hal-01925452 \\ https://hal.science/hal-01925452}

Submitted on 27 Nov 2018

HAL is a multi-disciplinary open access archive for the deposit and dissemination of scientific research documents, whether they are published or not. The documents may come from teaching and research institutions in France or abroad, or from public or private research centers.
L'archive ouverte pluridisciplinaire HAL, est destinée au dépôt et à la diffusion de documents scientifiques de niveau recherche, publiés ou non, émanant des établissements d'enseignement et de recherche français ou étrangers, des laboratoires publics ou privés. 


\section{LA DESTRUCTION DU PATRIMOINE CULTUREL À ALEP : BANALITÉ D'UN FAIT DE GUERRE ?}

Jean-Claude David, Thierry Boissière

\section{L'Harmattan | « Confluences Méditerranée »}

2014/2 N 89 | pages 163 à 171

ISSN $1148-2664$

ISBN 9782343040349

Article disponible en ligne à l'adresse :

https://www.cairn.info/revue-confluences-mediterranee-2014-2-page-163.htm

\section{Pour citer cet article :}

Jean-Claude David, Thierry Boissière « La destruction du patrimoine culturel à Alep : banalité d'un fait de guerre ? », Confluences Méditerranée 2014/2 (Nº 89), p. 163-171.

DOI 10.3917/come.089.0163

Distribution électronique Cairn.info pour L'Harmattan.

(c) L'Harmattan. Tous droits réservés pour tous pays.

La reproduction ou représentation de cet article, notamment par photocopie, n'est autorisée que dans les limites des conditions générales d'utilisation du site ou, le cas échéant, des conditions générales de la licence souscrite par votre établissement. Toute autre reproduction ou représentation, en tout ou partie, sous quelque forme et de quelque manière que ce soit, est interdite sauf accord préalable et écrit de l'éditeur, en dehors des cas prévus par la législation en vigueur en France. Il est précisé que son stockage dans une base de données est également interdit. 


\section{Jean-Claude David et Thierry Boissière}

Jean-Claude David est docteur en géographie ; chercheur associé au GREMMO (MOM, Lyon) ${ }^{1}$.

Thierry Boissière est docteur en anthropologie ; maître de conférences à l'université Lumière Lyon 2 et chercheur au GREMMO (MOM, Lyon); ex-responsable de l'antenne Ifpo d'Alep (2008-2010)2.

\section{La destruction}

\section{du patrimoine culturel à Alep : banalité d'un fait de guerre?}

Dans le conflit qui bouleverse actuellement la Syrie, les causes communautaires et confessionnelles sont régulièrement présentées comme étant les principales motivations des belligérants. La destruction du patrimoine, à Alep comme dans d'autres villes et sites archéologiques syriens, est l'une des conséquences les plus spectaculaires de ce conflit meurtrier, mais les causes de cette destruction sont multiples et les motivations idéologiques et confessionnelles ne sont peut-être pas les plus importantes. Pour comprendre l'acharnement destructeur dont Alep est la cible depuis 2012, nous proposons de revenir sur la nature et les origines historiques du concept de patrimoine tel qu'il est mis en oeuvre en Syrie et sur la perception que les habitants et les responsables en ont. Cette mise en perspective nous permet de mieux comprendre les réactions parfois hostiles ou indifférentes que l'on peut observer dans les deux camps, loyaliste et rebelle, vis à vis de ce patrimoine mondialement reconnu. 
J.-C. David et T. Boissière ont récemment dirigé la publication d'un important ouvrage sur Alep et ses territoires. Fabrique et politique d'une ville (1868-2011), Beyrouth, Presses de l'Ifpo, 2014.

T 'opinion mondiale s'émeut de la destruction du patrimoine en Syrie, comme ailleurs en d'autres guerres, presque autant que des violences faites aux populations. Le patrimoine est pourtant un objet ambigu, car l'émotion qu'il produit, aussi bien dans sa contemplation que par sa destruction, loin d'être universelle, dépend de la sensibilité spécifique du spectateur. Elle est marquée par l'éducation, les appartenances sociales et identitaires, certaines évolutions historiques. Or les listes du patrimoine sont généralement définies par les spécialistes, architectes ou historiens de l'art, suivant des critères esthétiques ou scientifiques qui peuvent être discutés. Avant d'analyser de plus près la destruction récente du patrimoine alépin, il est donc important de préciser ce qu'est le patrimoine dans ce contexte 3 .

\section{L'illusion d'universalité du patrimoine culturel en Orient}

La reconnaissance de la valeur de l'héritage culturel au ProcheOrient commence dès le XVII ${ }^{\mathrm{e}}$ siècle avec le regard des orientalistes, voyageurs, écrivains, savants, artistes. Les récits de ce « regard sur» sont plus largement diffusés et plus précis au XVIII ${ }^{\mathrm{e}}$ siècle. Au XIX ${ }^{\mathrm{e}}$ siècle, ils adoptent un parallélisme chronologique très proche de la prise de conscience du patrimoine en Occident, proximité qui se comprend quand on constate que les catégories de patrimoine reconnues par les orientalistes sont d'abord les vestiges de l'antiquité grecque et romaine, puis l'Égyptologie et les antiquités mésopotamiennes, tandis que le « Moyen-âge » Arabe et musulman ou chrétien n'a été pris en considération que plus récemment, souvent pour des raisons religieuses dans lesquelles les savants biblistes ont joué un rôle essentiel. Au $\mathrm{XX}^{\mathrm{e}}$ siècle, l'architecture, le tissu urbain, les monuments, deviennent objets d'une recherche scientifique et systématique. L'historien Jean Sauvaget est l'un des premiers pour Alep et Damas à mettre au point une démarche qui utilise à la fois les textes anciens, notamment en arabe, et les témoins matériels, et qui s'intéresse à l'histoire sociale autant qu'à la Grande histoire. Mais Sauvaget, imprégné de l'idéologie dominante de son époque, découvre et décrit ce patrimoine, qu’il participera à 
classer, tout en le dénigrant au profit des héritages antérieurs à l'islam. Sauvaget et d'autres chercheurs de l'époque mandataire ont cependant joué un rôle essentiel par leur recours systématique aux sources arabes, aux textes des chroniqueurs ${ }^{4}$, qu'ils ont largement contribué à faire reconnaitre.

\section{La fabrication du patrimoine à Alep et l'inscription dans le Patrimoine Mondial}

La sélection des objets patrimonialisés et les processus de patrimonialisation récents et actuels en Syrie, restent très marqués par des choix faits par d'autres que les habitants des lieux concernés, notamment à l'époque coloniale, conduisant progressivement à des dérives qui marient patrimoine et tourisme pour le meilleur et pour le pire. Le centre historique d'Alep a ainsi été inscrit dans la liste du Patrimoine Mondial en 1986, après un processus qui a commencé en juin 1978, date de la première demande d'inscription par le Ministère de la Culture. Comme ailleurs, la population n'a pas été consultée et les premières mesures de classement de quartiers en 1974 ont été considérées comme des formes d'expropriation déguisées, des privations de la jouissance intégrale d'un bien. En effet, la protection du patrimoine passe par le blocage de l'évolution normale de l'objet classé, le plus souvent tissu urbain constitutif des quartiers ou monuments d'architecture. Ainsi, l'application stricte des règles de protection entrave l'évolution normale de l'espace urbain et domestique, évolution nécessaire pour son adaptation aux changements du mode de vie, tandis que les mêmes lois sont souvent contournées, c'est le cas à Alep, pour permettre la transformation de la fonction du lieu, d'espace professionnel, artisanal, commercial, ou domestique, en espace touristique (hôtel, restaurant). La jurisprudence héritée des périodes les plus anciennes du droit musulman considère que la propriété privée et sa jouissance prime tout, avec pour seule limite le droit équivalent du voisin. L'expropriation même pour le bien commun était historiquement très peu pratiquée et réprouvée et son adoption pour la réalisation de divers projets modernes est un bouleversement encore difficile à faire admettre si ce n'est par la contrainte d'un pouvoir fort. Et finalement la reconnaissance de l'héritage matériel comme patrimoine culturel passe beaucoup par une incitation liée à la valorisation par des projets touristiques. Ces projets s'adressent aux touristes étrangers et nationaux et permettent souvent la réalisation d'un capital par la vente du bien immobilier et l'achat d'un 
logement dans un quartier répondant aux caractéristiques actuellement recherchées, suivant une évolution qui s'est accélérée au cours des trente dernières années. Cette évolution contribue au bouleversement des conditions sociales dans les quartiers anciens, malgré les directives de GTZ ${ }^{5}$ ou de l'UNESCO dont l'un des objectifs est de conserver ces quartiers avec leurs habitants et leurs activités d'origine. Des études montrent pourtant l'importance de l'espace domestique comme espace sacré de la famille et confirment l'importance de l'architecture domestique et des formes spatiales, non pas comme un acquis hérité et immuable mais comme le fondement d'évolutions et d'adaptations qui font de cet héritage l'inverse d'un patrimoine arrêté en contemplé selon la théorie de Françoise Choay ${ }^{6}$. S'il existe une reconnaissance du patrimoine par les alépins, c'est dans la revendication de leur espace domestique et surtout dans la liberté de le faire évoluer en fonction de leurs besoins, dans certaines limites ${ }^{7}$.

\section{Le conflit de 1979-1982 à Alep et Hama : guerres contre les villes et destruction du patrimoine}

Au cours des conflits des années 1978-1983, multiformes mais qui ont touché particulièrement Hama (avril 1981, février 1982) et Alep (depuis juin 1979), bien que de façons différentes, les objectifs étaient clairs : le régime est intervenu dès 1977 à Alep pour préparer l'ouverture de la ville ancienne par des percées lui permettant de procéder à des actions préventives contre les groupes de l'opposition armée, notamment les Frères Musulmans ; il est ensuite intervenu à Hama pour réprimer une insurrection violente en cours au printemps 1982. Dans les deux sites, les actions sont contre la ville et précisément contre ce qui en constitue le patrimoine. Les destructions prévues mais très partiellement réalisées à Alep dans le cadre de projets d'urbanisme, et les destructions considérables suite aux actions armées à Hama étaient le fait de la société de travaux publics de l'armée (Isken al-Askari) dans le premier cas et de l'armée dans le second. Les destructions à Hama se sont soldées par des dizaines de milliers de morts et la disparition du quartier Keilani, des norias qui s'y trouvaient et des monuments proches, la destruction partielle du Palais Azem, tandis que les menaces sur certains quartiers d'Alep n'ont été que partiellement exécutées. 
Si les destructions à Hama en 1982 étaient le fait de la répression violente d'une révolte, à Alep les nombreuses destructions dans les quartiers anciens à la même époque (15 hectares entre Bab al Faraj, l'ancien quartier juif, et Bab Neirab), étaient justifiées par des projets d'urbanisme et un idéal de développement et de modernité. Mais les unes et les autres étaient aussi fondamentalement politiques, et visaient, avec la destruction matérielle des quartiers, à détruire ou à tout le moins réduire la société qui y vivait. Quel que soit le contexte de la destruction, fait de guerre ou opération d'urbanisme, pour en comprendre le sens, il importe de connaître aussi bien ce qui est détruit que ce qui sera construit (ou ce qui est effectivement reconstruit : un jardin public et un hôtel Cham à Hama, un centre culturel et l'hôtel Sheraton sur le site de Bab al-Faraj à Alep). Le refus de classer la vieille ville d'Alep par l'ICOMOS en avril 1981 (classement « différé») est justifié alors par les destructions prévues de 45 hectares par des projets d'urbanisme. Les réactions d'une petite intelligentsia alépine et son action tant auprès des autorités que de la population (expositions de photos, affichage en ville, colloque international en 1982) aboutissent à la mise en sommeil des projets de percées à Jdeidé et à $\mathrm{Bab}$ al-Faraj, mais pas de ceux de Bab Neirab qui seront réalisés. Jusqu'en 2011 le mouvement en faveur de la conservation et de l'aménagement de la ville ancienne d'Alep suit son cours et évolue dans un sens relativement positif, d'intégration à la ville et à son centre, avec certaines fonctions spécialisées mais ponctuellement aux dépends de son caractère populaire.

\section{La guerre depuis juillet 2012 à Alep : les quartiers anciens et le patrimoine victimes collatérales de la guerre de guérilla ou/et objectifs idéologiques}

Au début de la guerre à Alep, certains murs de la ville portaient l'inscription "Alep nous te détruirons ». C'est effectivement ce qui se passe, essentiellement dans les quartiers anciens et les quartiers populaires informels, alors que la ville résidentielle moderne, inféodée au régime, reste épargnée. Les auteurs de ces graffitis de menaces contre la ville sont difficilement identifiables car la véritable force de destruction de la ville et de son patrimoine est la guerre elle-même qui oppose les deux principaux belligérants, l'armée nationale, relativement 
faible sur le terrain, à des groupes de guérilla installés dans les quartiers, les maisons, chez les gens, dans les monuments. La réplique de l'armée du régime est l'utilisation de l'aviation pour des bombardements divers, notamment avec les barils de TNT, qui «nettoient» le terrain, avec un minimum de risque pour l'armée et un maximum de pertes civiles : l'objectif, comme dans toute guerre urbaine dans un site difficilement accessible (Casbah, Médina, quartiers informels), est de détruire l'espace et la société qui servent de réduit et de soutien à la guérilla : la table rase est systématique et impressionnante. Il s'agit aussi, et le caractère aléatoire des frappes en témoigne, de punir les populations des quartiers qui ont accueilli les groupes rebelles.

Le patrimoine est détruit ponctuellement ou par zones plus larges au cours de combats localisés, de bombardements ou enfin par actions de sabotages. Ainsi, la destruction en 2012 de l'hôtel et restaurant Dar Zamariya, dans le vieux quartier chrétien de Jdeydé, se passe en plusieurs étapes : une très belle habitation du XVIII ${ }^{\mathrm{e}}$ siècle de taille moyenne, autrefois habitée par la famille chrétienne Zamariya, a été transformée en investissement touristique, avec un hôtel luxueux et un restaurant très fréquenté par la bourgeoisie surtout musulmane, opération réalisée par la famille Martini, notables sunnites, comptant un ancien mohafez au temps du mandat et un ministre sous Hafez al-Assad. L'hôtel/restaurant occupé comme QG par un groupe de rebelles, est attaqué par l'armée du régime dans une opération de récupération du terrain. Le résultat de la première phase est photographié et les photos diffusées par internet sont alors visibles sur différents sites (diffusion datée du 26 septembre 2012). La bataille au sol doit avoir opposé des groupes équipés d'armes légères : les lieux dans leur ensemble ont été dépouillés de tout décor, notamment les boiseries historiques de la qa'a et de l'ïwan/loggia ne sont plus là, mais les murs, les poutres des plafonds, sont presque intacts et notamment le décor extérieur des façades en pierre. Une autre série de photos diffusée peu après (diffusion datée du $1^{\mathrm{er}}$ octobre 2012) montre, dans les mêmes lieux, le gros oeuvre et le décor de pierre totalement détruits par des explosifs et cela jusqu'au ras du sol'.

La destruction d'une partie des souks semble aussi réalisée en plusieurs étapes, depuis octobre 2012 : les images satellites montrent plusieurs plages de destructions successives dans lesquelles les bâtiments sont détruits avec disparition des couvertures : d'autres photos montrent des souks où tout ce qui est en bois est incendié tandis que l'architecture est conservée. Des séquences vidéo montrent 
des groupes rebelles installés dans des galeries de souk non détruites mais incendiées et pillées.

Du point de vue de la destruction du patrimoine, trois phases sont donc repérables sans que l'on puisse les attribuer de manière certaine à une armée ou l'autre, phases qui ne se sont pas nécessairement toutes réalisées partout : pillages et récupérations, incendie et destruction au sol, bombardements ou dynamitage avec destruction des bâtiments.

Un autre aspect particulier qui pose la question des visées idéologiques des destructions du patrimoine, est celle qui semble viser systématique des bâtiments de la fin de l'époque ottomane répartis autour de la citadelle : l'ancienne municipalité ottomane qui avait été utilisée comme bureaux des passeports et visas (photos diffusées en février 2013), l'ancienne banque devenue le restaurant « Beroïa », ancien nom grec d'Alep, restaurant sans alcool très fréquenté par la bourgeoisie sunnite, l'ancien siège du Mufti, bâtiment baroque ottoman typiquement alépin et enfin l'hôtel Carlton, ancien hôpital national ottoman transformé en hôtel de luxe puis occupé par l'armée nationale, dynamité en mai 2014 par l'opposition au moyen d'un tunnel rempli de dynamite : l'explosion très spectaculaire a été largement diffusée par internet dans une vidéo de qualité professionnelle prise depuis deux sites : le dynamitage prévu et minuté était mis en scène comme un spectacle ${ }^{10}$.

La destruction du minaret du $\mathrm{XI}^{\mathrm{e}}$ siècle de la Grande Mosquée le 24 avril 2013, réprouvée par tous et non revendiquée, pourrait être la conséquence des tirs des deux armées sur un bâtiment dominant vraisemblablement occupé par des francs tireurs mais qui servait aussi à certains pour montrer leur habileté au tir. Ces actions diverses, suivies ou précédées de pillages, peuvent être des destructions intentionnelles ou des conséquences des combats et le plus souvent les deux à la fois. La destruction, quand elle est intentionnelle, peut viser des aménagements touristiques dont le luxe semble une provocation au regard de la pauvreté et des difficultés des habitants et des combattants issus des quartiers périphériques, mais l'hôtel Sheraton de Bab al-Faraj n’a pas été dynamité malgré son luxe et sa modernité écrasantes. Certaines agressions exceptionnelles ont visé un groupe ou une expression religieuse, comme les incendies et les pillages d'églises qui ont eu lieu au début de la guerre (au Midan, à Jdeydé, etc. ) et qui sont soit des provocations du régime et de ses milices soit des actes des rebelles au cours de leur action pour occuper des quartiers stratégiques réticents. 


\section{Conclusion}

Dans la destruction du patrimoine, les motivations politiques, idéologiques et culturelles, ciblées, sont rarement évidentes, mais peuvent exister de façon diffuse. La destruction du patrimoine, au sens ancien de biens hérités, pratique indissociablement liée à la guerre, est irréfutable. Il s'agit, en détruisant l'espace matériel de la vie quotidienne, d'entraver la perpétuation des familles, d'interrompre les généalogies, de punir, de pousser à la désorganisation et à l'exode, de couper les racines, comme dans la campagne on coupe les oliviers, on incendie les maisons, on détruit les récoltes, les provisions, les greniers, pour rendre difficile ou impossible le retour. Enfin les destructions conséquences de la guerre par bombardements contre la guérilla dans ces quartiers impénétrables pour les chars et autres véhicules d'artillerie sont évidentes aussi. Il est cependant difficile de distinguer ce qui appartient à telle ou telle motivation et est imputable à l'un ou l'autre camp. Cette guerre est une guerre à la ville et à la vie et par là une guerre au patrimoine le plus essentiel et le plus ordinaire, celui des boutiques et des espaces d'approvisionnement, du quartier et de la ruelle partagés par toute une société de voisinage et enfin de la maison, bien plus qu'au patrimoine culturel institutionnel : la ville, espace de vie, n'est pas patrimoine de la même façon qu'un site archéologique ou des objets dans un musée. La destruction par la guerre touche ainsi plus gravement le tissu domestique, économique et l'espace public populaire : en ce sens la cour de la Grande Mosquée est un espace plus essentiel pour les familles alépines que le minaret détruit. Les souks anciens étaient concurrencés par le développement de nouvelles centralités économiques mais ils restaient fréquentés par les populations plus pauvres de la ville ancienne, des quartiers populaires souvent conservateurs, ainsi que par les touristes épris de pittoresque. C'est l'ensemble de ce dispositif ancien, à la fois matériel et immatériel, et dans lequel s'inscrivait fortement le quotidien des gens, qui est ciblé et détruit selon des logiques qui laissent à penser que c'est une tout autre ville qui verra sans doute le jour après la guerre, et qu'elle ne se reconstruira pas sur les mêmes bases et les mêmes logiques anciennes et héritées, même si l'architecture est partiellement reconstituée. 
Notes :

1. Les recherches qu'il a menées sur les villes en Syrie, notamment Alep, depuis 1967, sont d'abord le produit de sa participation à l'élaboration du plan directeur de 1974, à la municipalité. Par la suite il a participé à l'instauration du premier plan d'aménagement et de conservation de la ville ancienne. Ses thèmes récurrents sont les espaces publics, l'espace domestique, le souk, le patrimoine matériel et immatériel, les architectures urbaines.

2. Les recherches qu'il mène sur la Syrie depuis 1990 ont porté sur l'agriculture urbaine, les stratégies sociales liées à la pauvreté et sur les espaces et les pratiques du commerce.

3. Cf. David Jean-Claude et Boissière Thierry (dir.), Alep et ses territoires. Fabrique et politique d'une ville (1868-2011), Beyrouth, Presses de l'Ifpo, 2014.

4. Pour Alep, Ibn Chaddad au XIII ${ }^{\mathrm{e}}$ siècle et ses prédécesseurs dont les écrits sont perdus, Ibn al-Chihna et Ibn al-Ajami au XV siècle et beaucoup d'autres jusqu'à Tabbakh et Ghazzi sous le Mandat et al-Asadi dans la seconde moitié du XXe siècle.

5. GTZ (Deutsche Gesellschaft für Technische Zusammenarbeit) : organisme allemand pour le développement qui a fait les études et réalisé les opérations de mise en valeur des quartiers classés patrimoine syrien et patrimoine mondial à Alep.

6. Choay Françoise 1970, L'allégorie du patrimoine.

7. Voir Moutia Touria 2014, "Patrimoine institutionnel et patrimoine vivant : le patrimoine habité », dans David et Boissière 2014, p. 445-479.

8. David Jean-Claude 1984 « Projets d'urbanisme et changements dans les quartiers anciens d'Alep », in Métral et Mutin 1984 Politiques urbaines dans le Monde Arabe, 351-365.

9. Voir par exemple : http://archaeolife.blogspot.fr/2012/10/photos-fromdestruction-of-dar-zamaria.html

10. http://www.youtube.com/watch?v=zJbeo5WE8tc 